\title{
EL HUMOR VERBAL EN NIÑOS DE EDUCACIÓN PRIMARIA: DESARROLLO DE LA CONCIENCIA METAPRAGMÁTICA
}

\author{
LARISSA TIMOFEEVA TIMOFEEV \\ Universitat d'Alacant \\ Grupo ACQUA (http://dfelg.ua.es/acqua) \\ Grupo GRIALE (http://dfelg.ua.es/griale)
}

Recibido: 15/09/2014

Aceptado: 24/11/2014

\section{Resumen}

Este trabajo constituye un estudio sobre el humor verbal en niños y niñas de $4 .^{\circ}$ curso de Educación Primaria (9-10 años). Tres grandes vías articulan el análisis. En primer lugar, el debate sobre las presuntas diferencias de género en la interpretación y el uso del humor verbal obtiene un interés especial en el caso de los niños, dado su menor nivel de «contaminación» por las convenciones y prejuicios sociales. En segundo lugar, se detecta la práctica ausencia de estudios sobre el humor infantil desde el punto de vista lingüístico, ya que el grueso de las investigaciones sobre el tema proviene de la psicología evolutiva. En tercer lugar, la etapa de edad objeto de este estudio responde a un periodo crucial en el desarrollo lingüístico y, especialmente, metalingüístico del niño.

Palabras clave: Humor infantil, conciencia metapragmática, perspectiva de género.

\begin{abstract}
This paper focuses on some aspects of humour acquisition, based on the analysis of humorous narratives written in Spanish by 9-10-year-old schoolchildren. The analysis is supported by three main ideas. Firstly, the debate about gender differences of humour processing becomes especially interesting in the case of children due to their less cultural and social «contamination». Secondly, an important lack of linguistic studies about children's humour is revealed since the main body of research is of
\end{abstract}


developmental psychology. Thirdly, the age period analysed in this paper is one of the most important in linguistic and, especially, metalinguistic development of children.

Keywords: Children's humour, metapragmatic awareness, gender perspective.

Feminismo/s 24, diciembre 2014, pp. 195-219 


\section{Introducción}

Este trabajo constituye una presentación general de un estudio sobre el humor verbal en niños y niñas de $4 .^{\circ}$ curso de Educación Primaria (9-10 años). Ha sido realizado en el marco del proyecto de investigación «Innovaciones lingüisticas del humor: géneros textuales, identidad y enseñanza del español» (FFI2012-30941) ${ }^{1}$.

La idea y el propósito del estudio obedecen a una serie de razones surgidas a raíz de la investigación previa sobre el tema. En primer lugar, el debate sobre las presuntas diferencias de género en la interpretación y el uso del humor verbal obtiene un interés especial en el caso de los niños, dado su menor nivel de «contaminación» por las convenciones y prejuicios sociales. En este sentido, nos parece esencial analizar si niños y niñas codifican de la misma manera sus enunciados humorísticos o muestran divergencias en lo que a las marcas e indicadores lingüísticos atañe.

En segundo lugar, nuestras indagaciones han revelado la práctica ausencia de estudios sobre el humor infantil desde el punto de vista lingüístico. Es sólida y relativamente prolija la investigación sobre el tema desde la psicología evolutiva que se dedica a la formación y el desarrollo de la competencia humorística en niños como parte de su maduración cognitiva y psicológica². En estos casos, el humor lingüístico se analiza como elemento integrante de la competencia global, pero nunca se observa desde su propia identidad. Por este motivo, un estudio como el que aquí presentamos se presenta innovador,

1. Este proyecto de investigación, dirigido por la Dra. Leonor Ruiz Gurillo, se lleva a cabo por el Grupo GRIALE (http://dfelg.ua.es/griale/). El estudio que se presenta fue coordinado por la Dra. Larissa Timofeeva, y su realización fue posible gracias a la ayuda y colaboración de los investigadores L. Aliaga, B. Alvarado, A. Mura, A. Pérez, S. Rodríguez y L. Ruiz.

2. cfr. McGhee, P. E. Humor: Its Origin and Development. San Francisco, W. H. Freeman, 1979; McGHEE, P. E. Understanding and Promoting the Development of Children's Humor. Dubuque, Kendall Hunt Publishing, 2002; MARTIN, R. A. The Psychology of Humor: An Integrative Approach. Burlington, Elsevier Academic Press, 2007; HOICKA, E. «The Pragmatic Development of Humor», en D. Mathews (ed.). Pragmatic Development in First Language Acquisition. Amsterdam, John Benjamins, 2014, pp. 219-238. 
pues pone el foco en la faceta lingüística del humor teniendo la psicología como telón de fondo. Nos interesamos por el aspecto «visible» del humor verbal, ya que son los elementos lingüísticos concretos, englobados bajo las denominaciones de marcas e indicadores del humor ${ }^{3}$, los que centran nuestra atención.

En tercer lugar, la etapa de edad objeto de este estudio -de 9-10 añosresponde a un periodo crucial en el desarrollo lingüístico y, especialmente, metalingüístico del niño ${ }^{4}$. Sus actuaciones verbales muestran cada vez menor grado de «cosificación», esto es, se hacen más abstractas e independientes de la presencia de los objetos en el entorno. Asimismo, la conciencia metapragmática se va perfilando y el discurso infantil va incorporando contenidos inferenciales acordes con el contexto lingüístico y extralingüístico.

Todos estos aspectos aparecen imbricados en el complejo fenómeno del humor verbal infantil. En lo que sigue, propondremos algunas líneas para avanzar en su investigación. Para ello, en primer lugar explicaremos los fundamentos teóricos de nuestro estudio (§ 2) para, a continuación, presentar la estructura (§ 3) y algunos resultados provisionales del mismo (§ 4). Unas breves conclusiones ( $\$ 5$ ) cerrarán el trabajo.

\section{Fundamentos teóricos}

Como hemos comentado, los niños que participaron en el estudio que exponemos aquí presentan un perfil de investigación muy interesante. Tal interés se apoya en, al menos, tres pilares teóricos. Por un lado, el desarrollo de la competencia metapragmática centra nuestra atención, pues los niños de la edad estudiada se encuentran en un momento clave marcado por el paso desde una comunicación epipragmática hacia una nueva fase caracterizada por la capacidad de, no solo ajustar su mensaje al contexto sino también de explicar y explicitar conscientemente sus decisiones pragmáticas. Por otro lado, dentro de dicha habilidad metapragmática, la competencia humorística experimenta un cambio sustancial en la franja vital estudiada en que el lenguaje empieza a adquirir un papel fundamental. Finalmente, en tercer lugar, el desarrollo de la habilidad escrita se presenta como un aspecto esencial

3. Para más detalle vid. Ruiz GuriLlo, L. La lingüística del humor en español. Madrid, Arco/ Libros, 2012, pp. 78-86.

4. Piaget, J. El lenguaje y el pensamiento del niño pequeño. Barcelona, Paidós, 1987; GoMBERT, J. «Metalinguistic development in first language acquisition», en L. Van Lier \& D. Corson (Eds.), Encyclopedia of Language and Education. Volume 6, Knowledge about language. Dordrecht/Boston, Kluwer Academic Publishers, 1997, pp. 43-51. 
dadas las características de nuestra investigación. A continuación presentaremos sucintamente los puntos clave de cada uno de los tres ámbitos teóricos implicados.

\subsection{La conciencia metapragmática}

El uso del lenguaje con fines comunicativos se fundamenta en un conocimiento consciente sobre el funcionamiento del sistema lingüístico y las relaciones que este establece con el contexto. Dicho de otro modo, todo acto verbal presupone un "control intencional de los parámetros lingüísticos que determinan la eficiencia del mensaje, en concordancia con aspectos extralingüísticos de la situación en la cual el enunciado se presenta» $»^{5}$. La conciencia metapragmática se entiende, por tanto, en relación a dicho control o capacidad del hablante de organizar su mensaje lingüístico de la manera que consiga inducir a su interlocutor hacia una respuesta perlocutiva buscada ${ }^{6}$.

Según defiende Verschueren ${ }^{7}$, la conciencia metapragmática se convierte en una noción imprescindible para poder entender nuestro comportamiento lingüístico:

Reflexive awareness is so central that all verbal communication is selfreferential to a certain degree, or that there is no language use without a constant calibration [...] between pragmatic and metapragmatic functioning. This phenomenon forms the proper domain of metapragmatics ${ }^{8}$

A pesar de su evidencia, la conciencia metapragmática no deja de ser una noción demasiado abstracta ${ }^{9}$. En palabras de Verschueren ${ }^{10}$, «awareness is not measurable, and the notion lends itself to easy speculation». Por eso, un estudio sobre los aspectos metapragmáticos obtiene su concreción en el análisis de las diversas pistas verbales utilizadas por los interlocutores que evidencian la conciencia reflexiva subyacente a la comunicación lingüística.

La conciencia metapragmática se adquiere en la infancia y es producto de nuestra educación comunicativa. Las circunstancias y las experiencias

5. CRespo, N. y Alvarado, C. «Conciencia metapragmática y memoria operativa en niños escolares». Literatura y Lingüística, 21 (2010), p. 95.

6. cfr. PortolÉs J. Pragmática para hispanistas. Madrid, Síntesis, 2004, pp. 31-46.

7. VERSCHUEREN, J. «Notes on the role of metapragmatic awareness in language use», Pragmatics, 10:4 (2000), pp. 439-456.

8. Ibíd., pp. 445-446.

9. cfr. GOMBERT, J. «Metalinguistic development in first language acquisition», p. 44; STUDE, J. «The acquisition of metapragmatic abilities in preschool children», en Bublitz, W. \& Hübler, A. (eds.), Metapragmatics in use. Amsterdam, John Benjamins, 2007, pp. 199-220.

10. VERSCHUEREN, op. cit., p. 445.

Feminismo/s 24, diciembre 2014, pp. 195-219 
verbales que rodean al niño durante su infancia perfilan su conocimiento sobre el lenguaje y le capacitan para reflexionar y expresar sus reflexiones sobre los comportamientos lingüísticos propios y de otras personas.

De acuerdo a esta visión, se destacan tres fases en la adquisición de la competencia metapragmática: de adquisición inicial, la epipragmática y la metapragmática ${ }^{11}$. En la primera fase de adquisición inicial el niño aprende a utilizar las primeras formas lingüísticas relacionadas de manera unívoca con determinados contextos. En la fase epipragmática el infante toma conciencia de que la relación entre lo lingüístico y lo contextual es más heterogénea, pero todavía no discrimina entre ambas informaciones. El ajuste de las formas lingüísticas a las circunstancias contextuales, por tanto, se realiza de manera automática, con consiguientes fallos ocasionales especialmente cuando tal ajuste se basa en una relación no literal. En esta fase epipragmática el niño todavía no sabe explicitar verbalmente sus decisiones pragmáticas. Es a partir de aproximadamente seis años cuando se aprende a discernir entre el mensaje lingüístico y el contexto, así como a ejercer un control sobre la relación que establecen ambas informaciones en el uso. El niño adquiere una competencia metapragmática que, además, le capacita para explicar explícitamente cómo realiza dicho control, tanto desde el punto de vista productivo como interpretativo.

\subsection{El humor}

Dentro de la competencia metapragmática en general, la expresión lingüística del humor constituye una de las habilidades más complejas. En este apartado haremos una breve revisión de los aspectos más relevantes del proceso de adquisición del humor en niños (2.2.1.) y del estudio del humor infantil desde la perspectiva de género (2.2.2.).

\subsubsection{Adquisición del humor}

La evolución de la competencia humorística transcurre imbricada en el desarrollo cognitivo general del niño y va progresando acorde a las diversas fases de maduración. Si bien los bebés protagonizan sus primeras actuaciones humorísticas -manifestadas a través de risa o sonrisa- a la temprana edad de 6 meses, el proceso global no se completa hasta los 10-11 años. A partir de esa edad se considera que el niño ya posee todas las herramientas cognitivas y lingüísticas para interpretar y producir el humor.

11. Gombert, J. Metalinguistic development. New York, Wheatsheaf, 1992. 
Diversos estudios apuntan a que hay dos ingredientes básicos cuya comprensión articula la formación de la competencia humorística en niños: la incongruencia y la intención. La incongruencia se relaciona con la sorpresa que nace de la discrepancia con las expectativas existentes respecto a un objeto, un hecho, etc. Como señala Cunningham ${ }^{12}$, se ha observado que ya los bebés menores de 7 meses ríen notablemente más ante un estímulo espontáneo e inesperado que ante uno repetido y previsible, lo que indica que la noción de incongruencia empieza a formarse a partir de las primeras manifestaciones del humor en la vida del bebé.

En lo que se refiere a la intención, a la edad de 14-18 meses los niños se muestran capaces de distinguir entre acciones intencionadas y los errores no premeditados. Un poco más tarde, a partir de 25 meses, ya pueden discernir entre una broma y un error, basándose, precisamente, en su carácter intencionado o no. El valor de estos descubrimientos, según Hoicka y Gattis ${ }^{13}$, reside principalmente en el hecho de que a través del humor el niño comprende que detrás de una acción humana puede haber una intención, aun cuando dicha acción es errónea ${ }^{14}$. Esta idea entronca directamente con la formación de la competencia metapragmática, ya que la comprensión de la intención humorística -primero desde la perspectiva interpretativa y más tarde también desde la productiva-implica un control consciente de la relación que se establece entre la información verbal y no verbal explícita y el valor implícito del mensaje.

La formación de la competencia humorística en niños atraviesa varias fases evolutivas que van parejas a su maduración cognitiva y lingüística. En 1979 Paul McGhee propone un modelo de desarrollo del humor infantil basado en la correlación con las cuatro etapas del desarrollo cognitivo de Piaget $^{15}$. Tras una reformulación posterior ${ }^{16}$, el autor considera la existencia de 6 fases -incluido un periodo inicial de pre-humor- por las que atraviesa el niño en su desarrollo del humor:

12. Cunningham, J. «Children's humor», en W. G. Scarlett, S. Naudeau, D. Salonius-Pasternak, I. Ponte (eds.), Children's play. SAGE publications, 2005, p. 99.

13. HoICKA, E. y GATTIS, M. «Do the wrong thing: how toddlers tell a joke from a mistake», Cognitive Development, 23 (2008), pp. 180-190.

14. En este sentido vemos también un punto de encuentro con la pragmática básica de Grice según la cual, se infrinjan o no las máximas conversacionales que propone, el Principio de cooperación siempre regulará la comunicación humana (GRICE, P. H. Studies in the Way of Words. Cambridge, MA, Harvard University Press, 1989).

15. Piaget, J., op. cit.

16. MCGHEE, P. E. Understanding and Promoting the Development of Children's Humor.

Feminismo/s 24, diciembre 2014, pp. 195-219 
Stage 0: Laughter Without Humor, de 0 a 6 meses;

Stage 1: Laughter at the Attachment Figure, de 6 a 12-15 meses;

Stage 2: Treating an Object as a Different Object, de 12-15 meses a 2-3 años;

Stage 3: Misnaming Objects or Actions, de 2 a 3-4 años;

Stage 4: Playing With Words, de 3 a 5-6 años;

Stage 5: Riddles and Jokes; a partir de 6-7 años.

El grupo de edad que abordamos en nuestro estudio se situaría en esta última franja evolutiva de Riddles and Jokes. Durante este periodo, gracias al progreso de la capacidad metalingüística, el niño mejora el control metapragmático de sus producciones lingüísticas. Por otro lado, su capacidad de manejar en su mente varios esquemas cognitivos a la vez y de imaginar o prever los posibles resultados de sus acciones, sin que tenga que realizarlas, le enseña la reversibilidad del pensamiento, esto es, que las operaciones mentales pueden ser revertidas anulando, de esta forma, sus efectos ${ }^{17}$. Todos estos aspectos capacitan al niño a entender el humor basado en la ambigüedad, los dobles sentidos y otras formas más sofisticadas del pensamiento. Si al principio de esta etapa el infante se dedica a memorizar las distintas bromas y chistes basados en juegos de palabras (actuación epipragmática) con el fin de compartirlos con sus amigos y la gente de su entorno, poco a poco su madurez metapragmática motiva su creatividad para generar sus propias muestras humorísticas. Otro aspecto fundamental que caracteriza este estadio tiene que ver con la fase de resolución del acto humorístico. Martin ${ }^{18}$ comenta que varios estudios han apuntado hacia la idea de que los niños van evolucionando desde el humor basado exclusivamente en la incongruencia hacia el humor fundamentado en la incongruencia-resolución ${ }^{19}$, y la transición coincide más o menos con el último estadio de McGhee. Otras investigaciones han sembrado alguna duda al respecto señalando que los niños en estadios anteriores igualmente utilizan procedimientos resolutivos, pero de otra índole; y que los niños mayores, o incluso los adultos, siguen disfrutando del humor que contiene solo incongruencia. Sin embargo, lo que sí parece cierto es que la comprensión de una estructura humorística tradicional (propia de un chiste, por ejemplo), con la incongruencia que se resuelve al final, se adquiere en ese último estadio de Riddles and Jokes.

En lo que se refiere al comportamiento lingüístico, cabe suponer que en este estadio fluctuará entre las actuaciones más epipragmáticas y las ya

17. MARTIN, R. A., op. cit., p. 240.

18. Ibíd., pp. 241-243.

19. Para las diversas concepciones teóricas del humor y sus componentes remitimos al lector a Ruiz GuRILlo, L. Op. cit. 
propiamente metapragmáticas. Esto está acorde con lo reflejado por la propuesta de $\mathrm{McGhee}^{20}$. Como se observa, algunas franjas de edad pertenecen a varias etapas evolutivas a la vez, lo cual significa que el niño no «abandona» radicalmente el estadio anterior para pasar al siguiente, sino que "convive» en varios de ellos. De esta forma, por ejemplo, un niño que se encuentra en el estadio 4 puede seguir encontrando divertido el humor propio de las etapas anteriores, aunque tenga dificultades todavía para interpretar el humor característico del estadio 5 .

\subsubsection{El humor y la perspectiva de género}

Junto con las investigaciones sobre la adquisición del humor, los estudios que abordan el tema desde la perspectiva de género tienen ya cierta tradición en la psicología. No obstante, tal tradición no es para nada continuista, ya que los trabajos más recientes rompen con la visión anterior que se ha reconocido como sesgada y carente de fundamento científico. Se han dejado de lado las afirmaciones sobre la ausencia del sentido del humor en las mujeres ${ }^{21}$ y se ha dirigido mayor atención hacia el humor espontáneo que surge en los contextos de interacción naturales ${ }^{22}$. Esta nueva perspectiva ha permitido demostrar que no existen diferencias esenciales entre la producción humorística de hombres y mujeres, si bien cada sexo muestra ciertas preferencias cualitativas. Por ejemplo, hombres y mujeres muestran índices similares en el uso de bromas agresivas, especialmente en el grupo de las personas de su mismo sexo. No obstante, los hombres se identifican más con el humor hostil y las bromas «enlatadas», mientras que las mujeres optan por las anécdotas propias o de otras personas. Tal recurso a experiencias personales también es utilizado por las mujeres para consolar o ayudar a sus amigos a superar situaciones difíciles, lo que se enmarca dentro de la tendencia general que han mostrado las mujeres en recurrir en mayor medida que los hombres al humor para crear o mantener los lazos de solidaridad en el grupo ${ }^{23}$. Estas «preferencias» humorísticas se forjan ya en la infancia, como han puesto de manifiesto algunas investigaciones.

20. MCGHEe, P. E. Understanding and Promoting the Development of Children's Humor.

21. LAKOFF, R. Language and Woman's Place. New York, Harper \& Row, 1975.

22. MARTIN, R. A. Op. cit., pp. 147-150.

23. Ibíd., p. 149.

Feminismo/s 24, diciembre 2014, pp. 195-219 
En referencia a este tema, Lyon ${ }^{24}$ alude a un estudio de Socha y Kelly ${ }^{25}$ que muestra que hasta los 7 años las producciones humorísticas infantiles presentan un cariz más prosocial, mientras que a partir de dicha edad -y coincidiendo más o menos con el último estadio evolutivo de la clasificación de McGhee $^{26}$ - su humor se vuelve cada vez más antisocial. Desde el punto de vista de género, al parecer a esa edad los chicos generan más humor agresivo y hostil que las chicas, y estas, a su vez, son menos proclives a dar muestras públicas de su sentido del humor. No obstante, los investigadores apuntan que semejante interpretación obedece a la perspectiva de adultos (padres y profesores), y no ha sido corroborada por los propios menores. Dicho de otro modo, los niños y niñas participantes en los estudios no tienen la misma percepción, tanto en lo que se refiere a mayor agresividad del humor de los chicos como a la «timidez» humorística de las $\operatorname{chicas}^{27}$. Bajo este prisma se plantea, por ejemplo, la necesidad de definir adecuadamente las nociones de agresividad u hostilidad que manejamos en relación al humor infantil, pues ello contribuiría a su correcto tratamiento educativo ${ }^{28}$.

\subsection{La competencia escrita}

Como señala Verschueren ${ }^{29}$ "writing is a verbal activity involving a generally high degree of consciousness in the making of linguistic choices (in comparison to more spontaneous oral interaction)». Por tanto, la elaboración de un texto escrito exige por parte del niño un alto nivel de madurez metalingüística, y metapragmática en particular. Como señalan Crespo, Benítez y Pérez ${ }^{30}$, el infante ha de realizar una representación mental de una situación retórica del escrito, a saber, su tópico, su posible destinatario y el propósito del escrito. «Esta representación le permite suplir la carencia de un contexto empírico y perceptible, típico de la lengua oral $»^{31}$.

24. LYON, C. «Humour and the young child. A review of the research literature». Televizion, 19 (2006), pp. 6-7.

25. SOCHA, T. J. \& KellY, B. «Children making 'fun': humorous communication, impression management, and moral development». Child Study Journal, 24:3 (1994), pp. 237-252.

26. MCGHEE, P. E. Understanding and Promoting the Development of Children's Humor.

27. cfr. BERGEN, D. «Humor, play and child development», en Klein, A. (ed.). Humor in children's lives. Westport, Praeger, 2003; LyON, C. Op. cit.

28. LYON, C. Op. cit., p. 7.

29. VeRSCHUEREN, J. Op. cit., p. 444.

30. Crespo, N., Benítez, R., PÉREZ, L. «Conciencia metapragmática y la habilidad para producir narraciones escritas», Revista Signos, 43:73 (2010), pp. 185-187.

31. Ibíd., p. 185. 
La franja de edad objeto de nuestro estudio está representada por escolares que han sido instruidos en la escritura durante al menos 4 años, desde que empezaron Educación Primaria. Por este motivo, su competencia escrita todavía no está plenamente asentada y era previsible que sus textos tuvieran muchos rasgos de oralidad al ser este el «terreno» que mejor conocen. De hecho, Crespo, Benítez y Pérez ${ }^{32}$ subrayan que en un estudio con niños de 8 años los participantes mostraron en sus narraciones bastante confusión en las secuencias temporales, pobreza de elementos cohesivos y otros rasgos que permiten concluir que los niños escriben como hablan.

En lo que se refiere a los tres componentes de la situación retórica -el tópico, el destinatario y el propósito- el primero, en el caso de nuestro estudio, fue suministrado por los propios investigadores, con el objetivo de no distraer a los niños en la búsqueda de un tema adecuado y de centrar su esfuerzo en el desarrollo del tema en sí. Esto nos permitía, además, homogeneizar las muestras y evitar que la elección más o menos acertada del tema determinara su carácter humorístico.

Por su parte, el componente del destinatario o lector del texto constituye uno de los aspectos más difíciles de manejar ${ }^{33}$. Como subrayan Crespo, Benítez y Pérez ${ }^{34}$, la tarea de elaborar el texto teniendo en mente a sus destinatarios se complica si estos no se encuentran en el contexto inmediato como pasa en la modalidad escrita. El niño, por tanto, ha de representarse mentalmente a su lector y atribuirle ciertas características, ya que ello determinará, por ejemplo, el registro que decida emplear. El joven escritor, consecuentemente, recurre a ciertas estrategias para suplir la ausencia de un lector claramente identificable, que pueden consistir en el empleo de esquemas, dibujos, acotaciones y explicaciones de todo tipo. Todos estos recursos pretenden clarificar aquellos aspectos susceptibles, en opinión del niño, de ser interpretados erróneamente y asegurar así la comprensión correcta del texto por parte del lector. En nuestro estudio lo único que sabían los escolares era que sus narraciones formaban parte de una investigación llevada a cabo por unos profesores de la Universidad de Alicante, aunque no parece que lo entendieran como información sobre los destinatarios de sus textos. Sin embargo, los niños sí que recurrieron a diversas técnicas de «creación» de su lector, tal y como veremos en el § 4 .

Respecto al tercer componente de situación retórica -el propósito- los participantes del estudio fueron informados que su tarea consistía en narrar

32. Ibíd., pp. 192-194.

33. cfr. CRESPO, N., BENÍTEZ, R. y CÁCERES, P. «La comprensión oral del lenguaje no literal y su relación con la producción escrita en escolares», Revista Signos, 40:63 (2007), p. 35.

34. Crespo, N., Benítez, R., Pérez, L. Op. cit., p. 186. 
una historia. Por tanto, el propósito del texto también fue acotado previamente lo que hizo que los niños optaran en su mayoría por la tipología narrativa, la más fácil para ellos, por otro lado.

\section{Estructura del estudio}

A continuación presentaremos la estructura del estudio, centrándonos en los objetivos, datos de los participantes, metodología y procedimientos, así como los aspectos básicos del procesamiento de datos.

\subsection{Objetivos del estudio e hipótesis de trabajo}

Tras presentar el andamiaje teórico que sustenta nuestro estudio, los objetivos que nos planteamos se pueden resumir según sigue:

- Observar la relación entre la expresión lingüística del humor y las fases de desarrollo del humor como competencia metapragmática en niños y niñas de 9-10 años.

- Analizar el uso de marcas e indicadores lingüísticos del humor por parte de niños y niñas a la edad estudiada en relación al nivel de desarrollo de su competencia escrita.

Respecto al primer objetivo planteado, acorde al modelo de $\mathrm{McGhee}^{35}$ los escolares de 9-10 años ya han alcanzado el último estadio de desarrollo del humor (Riddles and Jokes) donde el uso creativo del lenguaje es central. Asimismo, su competencia humorística está imbricada dentro del proceso de su maduración metapragmática que aún se está consolidando a esa edad. Crespo ${ }^{36}$ y Crespo, Benítez, Pérez ${ }^{37}$ muestran con datos empíricos que a los 8 años los niños todavía presentan una conciencia fundamentalmente epipragmática, por lo que en nuestro caso era esperable que el uso de procedimientos epipragmáticos aún fuera elevado. Asimismo, y dado el carácter no sistemático de la conciencia metapragmática, era previsible encontrar tanto las muestras epipragmáticas como las metapragmáticas dentro de las mismas narraciones.

Relacionado con lo anterior, Crespo, Benítez y Pérez ${ }^{38}$ subrayan la práctica ausencia de ejemplos del lenguaje figurado (ironía, fraseología, metáforas, hipérboles) en las narraciones de los niños de 8 años, lo que denota un nivel

35. McGHeE, P. E. Understanding and Promoting the Development of Children's Humor.

36. CRESPO, N. «La medición de la conciencia metapragmática de los niños: resolviendo la ambigüedad en la comprensión oral», RLA, 47:2 (2009), pp. 69-88.

37. Crespo, N., Benítez, R., Pérez, L. Op. cit.

38. Ibíd., p. 192. 
todavía bajo de desarrollo metapragmático. Teniendo en cuenta que nuestros informantes ya se encuentran en cotas algo superiores, y que la expresión del humor exige altas dosis de figuratividad, era esperable que la presencia de elementos figurados en las narraciones fuera mayor.

En referencia al segundo objetivo planteado, si bien el humor de los participantes en nuestro estudio se suponía más metapragmático, cabía esperar que la tarea de producir un texto escrito en un tiempo limitado iba a representar cierta dificultad. Ello se reflejaría en un notable apego a la oralidad, por lo que las marcas e indicadores del humor más frecuentes también serían más propios de la modalidad oral.

En lo que concierne a las diferencias de género, en el estudio citado de Crespo, Benítez y Pérez ${ }^{39}$ las niñas obtuvieron unas calificaciones significativamente superiores en su nivel de expresión escrita. Ello sugiere que si la tendencia se mantiene, las niñas de nuestro estudio presentarían una mayor diversidad en el uso de marcas e indicadores del humor. Por el contrario, si no existen demasiadas diferencias en este sentido, es posible que sea debido a que la brecha de la expresión escrita entre ambos sexos haya disminuido. El análisis de los datos obtenidos en el estudio también puso de manifiesto otros aspectos interesantes desde la perspectiva de género que se debaten en el $\$ 4$.

\subsection{Datos de los participantes}

El estudio que presentamos en este trabajo se llevó a cabo durante el curso académico 2012-2013 y en él participaron 149 alumnos de $4 .^{\circ}$ curso de Educación Primaria procedentes de cinco colegios -cuatro públicos y uno privadosituados en diversos puntos de la provincia de Alicante. Tras una revisión, la muestra se depuró hasta 148 narraciones. En la siguiente tabla recogemos los datos de los centros y la distribución de los participantes.

39. Ibíd., p. 194.

Feminismo/s 24, diciembre 2014, pp. 195-219 


\begin{tabular}{|c|c|c|c|c|}
\hline Centro & Tipo & Zona & $\begin{array}{l}\text { Programa } \\
\text { lingüístico }{ }^{40}\end{array}$ & \begin{tabular}{|c|}
$\mathrm{N}^{\circ}$ de \\
participantes
\end{tabular} \\
\hline DIVINA AURORA (Beneixama) & público & valencianoparlante & $\begin{array}{c}\text { Programa } \\
\text { d'ensenyament } \\
\text { en valencià } \\
(\mathrm{PEV})\end{array}$ & 13 \\
\hline $\begin{array}{l}\text { MARE DE DÉU DEL CARME } \\
\text { (La Canyada) }\end{array}$ & público & $\begin{array}{l}\text { predominantemente } \\
\text { valencianoparlante }\end{array}$ & $\begin{array}{c}\text { Programa } \\
\text { d'incorporació } \\
\text { progressiva } \\
\text { (PIP) }\end{array}$ & 10 \\
\hline ANTONIO MACHADO (Elda) & público & castellanoparlante & ----- & 41 \\
\hline $\begin{array}{l}\text { INMACULADA CONCEPCIÓN } \\
\text { (Torrevieja) }\end{array}$ & público & castellanoparlante & ----- & 48 \\
\hline $\begin{array}{l}\text { SAN ALBERTO MAGNO } \\
\text { (Monforte del Cid) }\end{array}$ & privado & castellanoparlante & \begin{tabular}{|c|} 
Programa \\
para el uso \\
progresivo \\
del inglés \\
como lengua \\
vehicular \\
(PUPILV); \\
francés o \\
alemán a partir \\
de $3^{\circ}$ de EP
\end{tabular} & 37 \\
\hline
\end{tabular}

1. Datos de los participantes

Como muestra la tabla, los resultados de nuestro estudio permiten una interpretación atendiendo a diversas variables, a saber, el sexo del alumno, el tipo de centro (público o privado), así como el entorno y el programa lingüísticos que sigue en su centro.

Respecto a la distribución por género, entre los 148 informantes 75 eran chicas y 73 chicos, lo que constituye $50,68 \%$ y 49,32\% respectivamente, tal y como recoge el siguiente gráfico:

40. Para más información sobre los programas PEV y PIP consúltese la web de la Conselleria d'Educació, Cultura i Esport de la Generalitat Valenciana http://www.cece.gva. es/ocd/sedev/es/pro_edu_bil.htm. Para saber más sobre PUPILV, diríjase a la web del Colegio San Alberto Magno http://www.sanalbertomagno.eu/proyectos-educativos/ idiomas.html. 


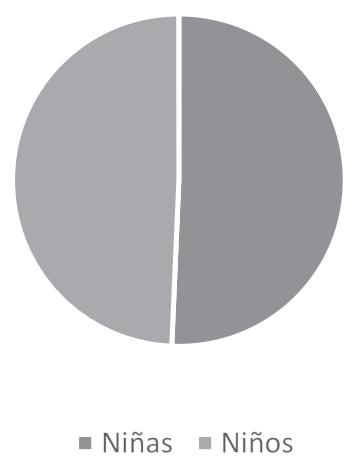

2. Distribución de informantes por género

\subsection{Procedimientos y metodología empleados}

La tarea que realizaron los participantes en el estudio consistía en una narración humorística con el siguiente tema:

\section{Un cuento humorístico}

IMAGÍNATE QUE EN EL MARCO DE UN PROGRAMA DE INTERCAMBIO ESCOLAR TIENES QUE PASAR DOS SEMANAS COMO ALUMNO DE UN COLEGIO EN... ¡MARTE!

Cuéntanos cómo es tu día de colegio, tus asignaturas, tus profesores, tus compañeros, qué hacéis en el patio, cómo te comunicas con tus amigos marcianos, cómo es el idioma en que hablas con ellos... ¡Seguro que es divertido!

\section{Tema de la narración}

El ejercicio era anónimo y lo único que tenían que indicar los informantes era su sexo, marcando la casilla correspondiente.

Como se observa, el enunciado de la tarea pretende acotar, tanto el tópico (colegio marciano) como el propósito de la situación retórica (narración humorística) (§ 2.3). La elección del tema, o tópico, obedece a los siguientes criterios:

- Que sea un tema cercano y conocido por todos los alumnos, independientemente de las características de sus centros;

- Que sea un tema lo suficientemente abierto para permitir a los participantes un amplio marco para la creación;

- Que sea un tema adecuado para construir un relato humorístico. 
De esta manera, el tema propuesto sitúa a los niños en uno de los ámbitos de donde proviene la mayor parte de ejemplos humorísticos de su vida ${ }^{41}$. Al mismo tiempo, el mundo escolar conocido por los participantes se traslada a otra dimensión planetaria, contexto que nos pareció susceptible de generar situaciones de incongruencia en las que basar la narración.

Respecto a la actuación de los profesores de los centros participantes, todos ellos se esforzaron en crear una predisposición positiva y despertar el interés de sus alumnos hacia la tarea.

\subsection{Procesamiento de datos}

Todas las narraciones recogidas fueron etiquetadas de acuerdo con el siguiente código: Centro/Sexo/Número correlativo, donde A marca que es niña y $\mathrm{O}$, que es niño. Así por ejemplo, el código SA5 indica que se trata de la narración realizada por una alumna del colegio San Alberto Magno correlativamente numerada como 5; IO16 se refiere a la narración número 16 de un alumno del colegio Inmaculada Concepción, etc.

El procesamiento de las narraciones desembocó en la creación de una base de datos Excel donde se recogen las distintas marcas e indicadores lingüísticos que utilizaron los niños y niñas en sus narraciones para expresar el humor. No obstante, esta fase del estudio también reveló una serie de deficiencias que serán subsanadas en el futuro, como por ejemplo, la ausencia de un marcador que mida las muestras epipragmáticas frente a las metapragmáticas, la carencia de un indicador del humor hostil o agresivo, o un reflejo más preciso en dicha base de datos de los elementos gráficos que usaron los informantes al describir sus intercambios lingüísticos con los alumnos «marcianos».

\section{Debate y análisis de algunos resultados}

En este apartado analizaremos algunos resultados del estudio en relación a los objetivos planteados.

\subsection{El humor y la conciencia metapragmática}

En nuestra hipótesis de trabajo planteábamos que para la franja de edad estudiada la presencia de muestras epipragmáticas en la expresión del humor aún sería notable. No obstante, el diseño inicial de nuestro estudio no contempló una herramienta de medición de este aspecto, por lo que en estos momentos nuestras consideraciones se basan en las impresiones generales de los

41. cfr. Bergen, D. Op. cit.; LyON, C. Op. cit. 
investigadores participantes. En este sentido, las narraciones humorísticas de los niños de $4 .^{\circ}$ de Primaria demuestran un nivel bastante alto de conciencia metapragmática ya que se hace un uso reflexivo del lenguaje. Uno de los ejemplos más claros de ello lo encontramos en la «lengua marciana» que inventan los informantes en sus relatos. Veamos algunos casos:

1. Cuando empezo a hablar la profesora me di cuenta que el idioma marciano era que las palabras estaban al revés por ejemplo de lengua=augenl. [...] Acabamos de comer y nos fuimos al patio y me encontre con el que hable y me dijo aloh arto zev em omall nairda [...] (SA5) ${ }^{42}$

2. Al día siguiente nos dimos cuenta que a las palabras les añadian una $u$ y si esa palabra tenia u se la quita van. Quando tocaron a mi puerta me pregunte: ¿Quién seria? Cuando abri la puerta era un marciano que decia: tuoma t niforme (SA7)

Como se observa, en estos ejemplos la invención del idioma sigue un determinado patrón que se adapta al contexto del relato humorístico. Todo ello indica una clara conciencia metapragmática de los jóvenes escritores que son capaces, no solo de realizar una reflexión sobre el lenguaje, sino de explicitarla a través de la invención de una lengua sistematizada. Asimismo, tal «juego» reflexivo del lenguaje con fines humorísticos es propio del último estadio de desarrollo del humor (Stage 5: Riddles and Jokes) según McGhee ${ }^{43}$, lo que confirma que nuestros informantes ya han alcanzado un alto nivel de control sobre el lenguaje y su uso para diversos fines comunicativos.

No obstante, dentro de este mismo tópico, es representativo el número de ejemplos que muestran una visión mucho más epipragmática, como sucede en 3:

3. [...] hablamos en el idioma marciano ola en marciano és coto, adiós es patarra, etc. (IO16).

La inventiva lingüística no parece seguir en este caso ninguna pauta claramente identificable. Más bien parece que lo único que guía al escritor es el absurdo fónico de los términos inventados. En otras palabras, este tipo de humor corresponde al estadio Playing With Words, en la que la construcción de la incongruencia se basa en procedimientos más epipragmáticos que no discriminan entre lo lingüístico y lo contextual y buscan solo la «sorpresa» visible o audible.

42. En todos los ejemplos se preserva la redacción y la ortografía originales.

43. MCGHEE, P. E. Understanding and Promoting the Development of Children's Humor. 
Las narraciones analizadas presentan más pruebas de la todavía fuerte impronta epipragmática, no solo en relación a la lengua inventada, como sucede en 4 y 5 :

4. las asignaturas que hacemos son las siguientes: Canguro, tonto, sombrilla en el medio, mapa (gorila gorigolia) (IA8)

5. En el nuevo colegio que voy me divierto mucho hay muchas asignaturas cómo oparetolxo qué es matemáticas, sotomarreplucos qué es lengua, casacorotamopatolimas que es cono, rasdatelaunatimosco qué es valenciá, rrrapatografitica qué es inglés, etc. (IO16)

No obstante, esto no significa que los escolares que participaron en el estudio se muevan todavía en el humor epipragmático. De hecho, en ocasiones dentro de la misma redacción encontramos ejemplos de la «convivencia» de ambas fases. Veámoslo en 6:

6. Mis asignaturas son muy divertidas tenemos: matemarcía, lenguina, educación martica, conocimiento del miedo, lecturma, mosico y ramarten (IO3)

En este caso, si bien la sistematicidad en la creación de los nombres de las asignaturas resulta algo confusa, la utilización reiterada de los segmentos ma o mar indica que el alumno busca conscientemente una conexión de sus creaciones léxicas con el contexto del «currículo» marciano.

Una combinación interesante la encontramos también en 7:

7. Para ir al colegio llevan mochilas coete asi es como las llamo yo porque en marciano es: mrmaques chuques. ¡A que esa palabra es rarísima! (IA5)

Se observa que la palabra inventada es incongruente meramente en su aspecto sonoro. No obstante, en el mismo enunciado a continuación la informante explicita una reflexión ( $i$ A que esa palabra es rarísima!) que muestra que existe una discriminación entre la incongruencia de la forma fónica y el contexto de una lengua inventada. De alguna manera, la apostilla que deja la niña actúa como un elemento resolutivo ante la incongruencia formal del término que inventa.

Esta adición de la fase de resolución también indica el tránsito hacia un humor más metapragmático. De hecho, desde el punto de vista estadístico hemos constatado que en 19 casos los informantes recurren a la estructura de incongruencia-resolución en sus producciones humorísticas. Si bien este número solo representa el 12,84\% del total, su presencia es reseñable. En este sentido, desde la perspectiva de género, cabe observar que de esas 19 narraciones 13 son de autoría femenina, lo que duplica el número de muestras masculinas (gráficos 4 y 5 ). 


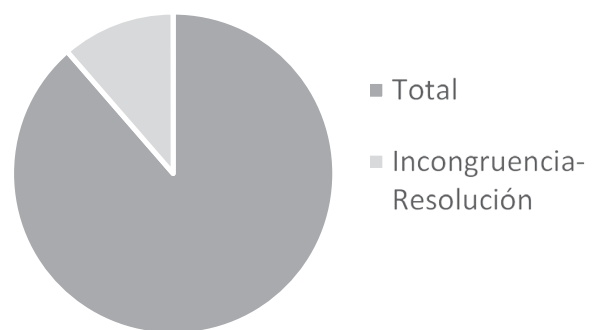

4. Incongruencia-Resolución en las narraciones infantiles

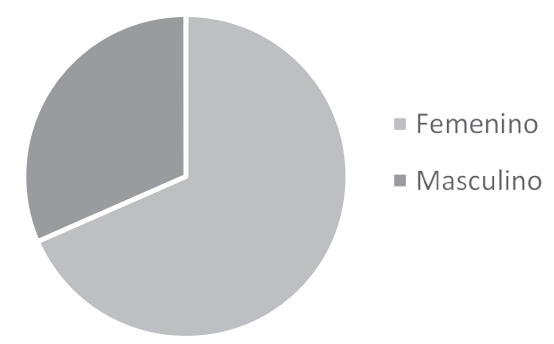

5. Incongruencia-Resolución: distribución por género

Otro indicador de que los escolares de nuestro estudio ya han alcanzado un importante nivel de conciencia metapragmática en su producción humorística es la presencia del lenguaje figurado. La hipótesis planteada al respecto (vid. § 3.1) de que sus índices serían mayores - por la edad de nuestros informantes y por la tarea encargada- se ha confirmado. En el siguiente gráfico se refleja la presencia de las muestras figuradas en las composiciones analizadas:
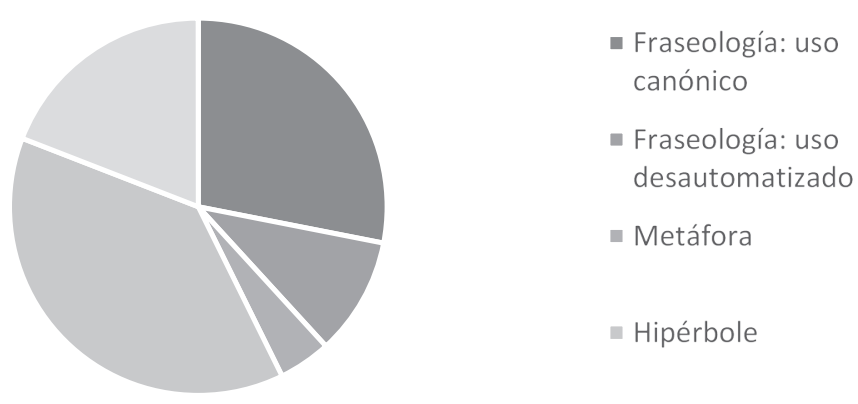

6. Lenguaje figurado

No se han observado diferencias notables en el uso de estos elementos entre chicos y chicas.

Para concluir este primera parte, podemos decir que la percepción de los investigadores es que las chicas, en general, muestran cotas ligeramente más altas de conciencia metapragmática que se reflejan en una mejor producción escrita y en un uso más variado de marcas e indicadores verbales del humor. No obstante, este aspecto necesita una comprobación empírica más enfocada y perfilada que esperamos acometer en el futuro. 


\subsection{El humor y la expresión escrita}

En lo que atañe a la expresión escrita, la mayor parte de los participantes de nuestro estudio han construido narraciones razonablemente coherentes, aunque con abundantes faltas de ortografía y bastantes carencias en el uso de elementos cohesivos. El uso de la puntuación ha sido más que deficiente lo que indica que la competencia escrita todavía se encuentra en plena formación y que muchos de los escolares a esa edad, a pesar de tener un aceptable control metasemántico y metapragmático, aún no son capaces de hacer una organización gráfica de su discurso oral mediante los signos de puntuación. En general, podemos decir que la tendencia de que los niños y las niñas escriben como hablan todavía es muy fuerte. La sensación común de los investigadores que participaron en el estudio es que las chicas presentan una escritura más cuidada, tanto en la letra como en la elaboración del texto, aunque esta sensación no fue comprobada estadísticamente.

Respecto a la representación de situación retórica, cabe señalar que dentro del tópico global que propusimos a los escolares también marcamos algunos posibles subtópicos. La construcción de las incongruencias se ha basado en algunos de ellos. Dentro de los mismos el «aspecto físico» de los marcianos ocupa el primer lugar, ya que aparece en el $66,89 \%$ de las composiciones. Lo siguen los subtópicos de «vida académica» $(60,14 \%)$, situaciones de «choque cultural» $(54,05 \%)$, «alimentación» $(24,32 \%)$ y «medios de transporte» $(19,59 \%)$. En el gráfico 7 reflejamos estos datos en números absolutos:

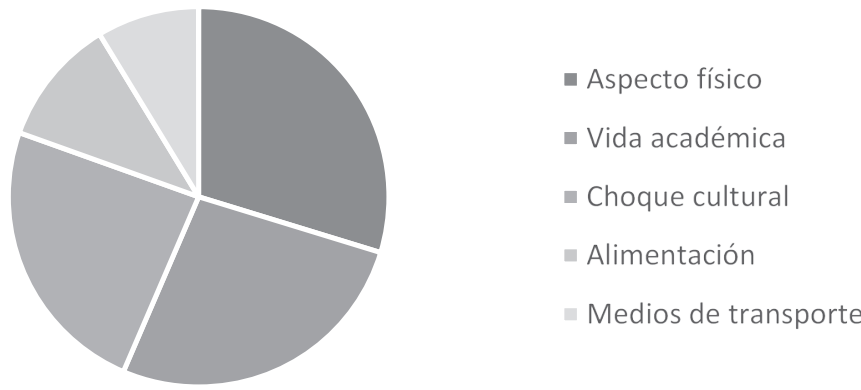

7. Distribución de subtópicos

En lo que concierne a la distribución por género, solo en los subtópicos de «alimentación» y de «aspecto físico» hay diferencias reseñables. En el primero, de los 36 casos recogidos 21 son de escritoras chicas, mientras que los chicos describen las costumbres alimentarias marcianas en 15 narraciones. En 
referente al subtópico de «aspecto físico», de los 99 casos 53 son de chicas y 46 de chicos. Si bien las diferencias no son demasiado grandes, llama la atención la preferencia de las informantes femeninas por el tema de la «comida marciana». En esta línea, una investigación secundaria podría analizar no solo las diferencias cuantitativas sino también las cualitativas en el tratamiento de estos dos subtópicos por parte de chicas y chicos.

En nuestro estudio hemos confirmado, además, que los chicos manifiestan un humor más agresivo. No habilitamos ningún instrumento de medición de este aspecto, pero hemos detectado que solo las narraciones masculinas contienen muestras de humor violento. Lo vemos, por ejemplo, en 8 donde el autor al describir a los marcianos ataca de manera agresiva su imagen:

8. [...] las gafa er de vichos ivan descalzos en el patio se tiran muchos pedo son super guaros cochinos asta se mean tiran los mocos los pedos los guardan duermen en el sue no san ven contar los números son tontos (IO11).

El análisis de los cinco indicadores del humor más usados por los escolares en sus narraciones revela otros aspectos interesantes. El gráfico 8 refleja estadísticamente tales indicadores:
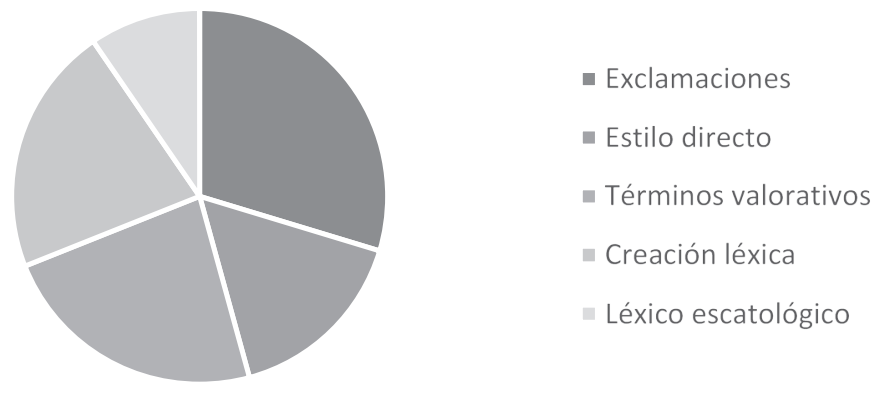

8. Cinco marcas e indicadores del humor más usados

Observamos que indicadores como «exclamaciones», «estilo directo» o «creación léxica» evidencian, de nuevo, que el apego a la oralidad es importante. Por su parte, la presencia del indicador «léxico escatológico»-propio sobre todo de los estadios del humor anteriores y que entre nuestros informantes supone 28 casos- concuerda con la idea de que los niños no abandonan drásticamente un estadio previo para pasar al siguiente, sino que «conviven» en varios de ellos a la vez. En lo que concierne a su reparto por género, el uso del léxico escatológico es solo ligeramente mayor en chicos (16 narraciones) 
que en chicas (12), dato que ha sido algo sorpresivo ya que esperábamos una mayor diferencia a favor de los chicos.

La difícil tarea de representarse mentalmente al destinatario de su texto lleva al niño a adoptar estrategias para «crear» a su lector y asegurarse así la correcta comprensión de su relato. Entre tales estrategias se encuentran explicaciones, precisiones o dibujos, entre otros elementos. El joven escritor se toma estas precauciones por no estar seguro de su control sobre la situación retórica de su relato lo que refleja su propia desconfianza en sus habilidades escritas. A continuación proponemos algunos ejemplos que ilustran claramente lo dicho. El primero, una parte del cual ya ha sido analizada arriba, contiene varias acotaciones que pretenden guiar al lector hacia una interpretación correcta de elementos humorísticos:

9. Para ir al colegio llevan mochilas coete asi es como las llamo yo porque en marciano es: mrmaques chuques. ¡A que esa palabra es rarísima! También tienen música y la más famosa es proma broma estail, es como ganga estail pero en marciano (IA5)

En 9 observamos claramente cómo la informante se encuentra muy pendiente de su posible lector. Primero, le invita a compartir su reflexión metalingüística sobre el carácter raro de la palabra marciana. A continuación, le aclara el significado de la pieza musical marciana. En suma, ante la imposibilidad de obtener un feedback inmediato, como sucede en la modalidad oral, la alumna adopta una actitud protectora con su potencial destinatario y le facilita la tarea interpretativa. Asimismo, tal y como ya veíamos en el § 4.1., estos elementos desempeñan la función de resolución frente a la incongruencia planteada por el léxico inventado.

Esto mismo sucede en 10, donde la informante «traduce», y por tanto resuelve, el nombre incongruente del colegio marciano para que su lector capte adecuadamente la intención humorística de su peculiar creación léxico-gráfica:

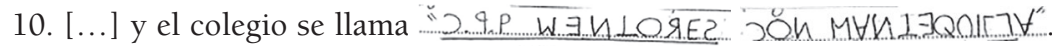
Sería, colegio público y privado «Mentores con mantequilla» (SA2)

En 11, a su vez, es el recurso de dibujo que desempeña la misma función aclaratoria y resolutiva:

11. Cuándo llegué al colegio en marte (llegue en nave) marte era gigante. Allí no habia nadie y era super raro porque en cuanto llegue ya me parecia tener alucinaciones, ví un marciano con tres ojos y tres pies, yo lo llamaría el triple-tres» (IO32)

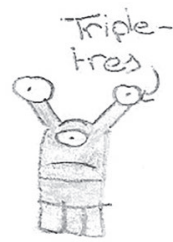


En todos estos ejemplos los autores de las narraciones buscan suplir la falta de inmediatez con el destinatario e intentan imaginar, «crear» a su lector al que proporcionan pistas interpretativas.

\section{Conclusiones}

En este trabajo hemos presentado un estudio sobre el humor verbal en niños y niñas de 9-10 años. La tarea se llevó a cabo con los alumnos de diversos centros de la provincia de Alicante y consistió en la escritura de una narración humorística sobre un tema dado. Nuestro objetivo general era observar el uso de marcas e indicadores lingüísticos del humor en relación al desarrollo de la conciencia metapragmática ${ }^{44}$ y de la competencia escrita. Asimismo, la perspectiva de género ha sido otro punto de atención.

Algunos de los resultados debatidos en este trabajo muestran que a la edad de 9-10 años los niños y las niñas ya manifiestan una conciencia metapragmática bastante alta, al menos en comparación con los resultados de los experimentos realizados con los niños de 8 años $^{45}$. Como consecuencia, el humor de nuestros informantes ya recurre al uso de marcas e indicadores lingüísticos propios del último estadio -Riddles and Jokes-del desarrollo del humor según $\mathrm{McGhee}^{46}$. No obstante, la presencia de procedimientos más epipragmáticos y propios de estadios del humor anteriores (sobre todo, del Playing With Words) es notable. Ello confirma que los niños no «abandonan» las etapas anteriores para pasar a la siguiente, sino que «conviven» en varios estadios.

Desde la comparación por género, en la franja de la edad analizada las niñas parecen mostrar una competencia metapragmática algo más alta y su humor se articula con mayor frecuencia en dos fases, de incongruencia y de resolución. Dicho esto, el dato necesita una comprobación más exacta que se abordará en investigaciones futuras.

Estas consideraciones están acordes con el análisis de la competencia escrita, donde las niñas por lo general han presentado narraciones más cuidadas. En términos globales, el peso de la oralidad sigue siendo notable. Solo en el caso de los varones se han detectado muestras del humor agresivo. Asimismo, las niñas han manifestado una curiosa preferencia por el subtópico de alimentación marciana.

44. GOMBERT, J. «Metalinguistic development in first language acquisition»; VERSCHUEREN, J. Op. cit.

45. vid. Crespo, N., Benítez, R., Pérez, L. Op. cit.

46. MCGHEE, P. E. Understanding and Promoting the Development of Children's Humor.

Feminismo/s 24, diciembre 2014, pp. 195-219 
En lo que se refiere a las marcas e indicadores del humor concretos, no se han detectado diferencias significativas entre niños y niñas. Sin embargo, esta comparación se tiene que abordar en un trabajo más particularizado y centrado, y no en una presentación global como esta.

\section{Referencias bibliográficas}

BERGEN, D. «Humor, play and child development», en Klein, A. (ed.). Humor in children's lives. A guidebook for practitioners. Westport, Praeger, 2003, pp. $17-32$.

CRESPO, N., BENÍTEZ, R. y CÁCERES, P. «La comprensión oral del lenguaje no literal y su relación con la producción escrita en escolares». Revista Signos, 40:63 (2007), pp. 31-50.

CRESPO, N. y Alfaro, P. «La conciencia metapragmática en la edad escolar». Boletin de lingüistica [online], 21:32 (2009). Disponible en: http://www.scielo. org.ve/scielo.php?script=sci_arttext\&pid=S0798-97092009000200002\&lng $=$ es\&nrm=iso

CRESPO, N. «La medición de la conciencia metapragmática de los niños: resolviendo la ambigüedad en la comprensión oral», RLA, 47:2 (2009), pp. 69-88.

CRESPO, N. y AlFARO, P. «Desarrollo tardío del lenguaje: la conciencia metapragmática en la edad escolar». Universitas Psychologica, 9:1 (2010), pp. 229-240.

CRESPO, N. y AlVARADO, C. "Conciencia metapragmática y memoria operativa en niños escolares». Literatura y Lingüística, 21 (2010), pp. 93-108.

CRESPO, N., BENíteZ, R., PÉREZ, L. «Conciencia metapragmática y la habilidad para producir narraciones escritas», Revista Signos, $43: 73$ (2010), pp. 179-209.

Cunningham, J. «Children's humor», en W. G. Scarlett, S. Naudeau, D. SaloniusPasternak, I. Ponte (eds.) Children's play. SAGE publications, 2005.

GOMBERT, J. Metalinguistic development. New York, Wheatsheaf, 1992.

GOMBERT, J. «Metalinguistic development in first language acquisition», en L. Van Lier \& D. Corson (Eds.), Encyclopedia of Language and Education. Vol. 6. Knowledge about language. Dordrecht/Boston, Kluwer Academic Publishers, 1997, pp. 43-51.

GRICE, P. Studies in the Way of Words. Cambridge, MA, Harvard University Press, 1989.

HoickA, E. «The Pragmatic Development of Humor», en Pragmatic Development in First Language Acquisition, Matthews, D. (ed.), Amsterdam, John Benjamins, 2014, pp. 219-238.

HoickA, E. y GatTis, M. «Do the wrong thing: how toddlers tell a joke from a mistake», Cognitive Development, 23 (2008), pp. 180-190.

LAKOFF, R. Language and Woman's Place. New York, Harper \& Row, 1975. 
LYON, C. «Humour and the young child. A review of the research literature». Televizion, 19 (2006), pp. 4-9. Disponible en: http://www.br-online.de/jugend/izi/ english/publication/publications-televIZIon.htm.

MARTIN, R. A. The Psychology of Humor: An Integrative Approach. Burlington, Elsevier Academic Press, 2007.

McGHEE, P. E. Humor: Its Origin and Development. San Francisco, W. H. Freeman, 1979.

McGHEE, P. E. Understanding and Promoting the Development of Children's Humor. Dubuque, Kendall Hunt Publishing, 2002.

Piaget, J. El lenguaje y el pensamiento del niño pequeño. Barcelona, Paidós, 1987.

PORTOLÉs, J. Pragmática para hispanistas. Madrid, Síntesis, 2004.

Ruiz Gurillo, L. La lingüística del humor en español. Madrid, Arco/Libros, 2012.

SochA, T. J. \& Kelly, B. «Children making 'fun': humorous communication, impression management, and moral development». Child Study Journal, 24:3 (1994), pp. 237-252.

STUDE, J. «The acquisition of metapragmatic abilities in preschool children», en Bublitz, W. \& Hübler, A. (eds.), Metapragmatics in use. Amsterdam, John Benjamins, 2007, pp. 199-220.

VERSCHUEREN, J. «Notes on the role of metapragmatic awareness in language use», Pragmatics, 10:4 (2000), pp. 439-456. 